\title{
ABUS SEXUELS D'ENFANTS : ASPECTS HISTORIQUES, JURIDIQUES ET PRÉJUDICE AU DÉVELOPPEMENT DE L'ENFANT
}

\section{RÉVISER L'ARTICLE}

GONÇALVES, Natamy de Almeida ${ }^{1}$, DIAS, Camila Santos²

GONÇALVES, Natamy de Almeida. DIAS, Camila Santos. Abus sexuels d'enfants : aspects historiques, juridiques et préjudice au développement de l'enfant. Revista Científica Multidisciplinar Núcleo do Conhecimento. An 06, Ed. 09, Vol. 01, p. 183 à 208. Septembre 2021. ISSN: 2448-0959, Lien d'accès: https://www.nucleodoconhecimento.com.br/psychologie-fr/aspects-historiques

\section{RÉSUMÉ}

Parce qu'il s'agit d'une violence qui sévit dans plusieurs enfants et adolescents dans le monde à travers l'histoire, l'abus sexuel d'enfants nécessite une attention particulière, étant donné qu'il s'agit d'un phénomène complexe et, dans de nombreux cas, difficile à identifier, étant l'agresseur généralement une personne de confiance de la famille et qui est censée prendre soin de la victime, comme parents biologiques, beaux-pères, oncles ou amis proches, par exemple. En outre, la plupart des cas d'abus sexuels se produisent au domicile de la victime, où l'on s'attend peu à ce que l'enfant et l'adolescent se trouvent dans une situation vulnérable. Sur la base des facteurs décrits, cette recherche présente la question directrice suivante : qu'est-ce que l'abus sexuel et quels sont ses impacts sur le développement de l'enfant? Cet article visait à passer en revue les aspects historiques et juridiques de l'abus sexuel, en explorant la complexité de ses définitions et en présentant les effets

\footnotetext{
${ }^{1}$ Diplômé en neuropsychologie de la Faculté de technologie, des sciences et de l'éducation - FATECE, diplômé en psychologie organisationnelle et du travail à la Fondation Hermínio Ometto et diplômé en psychologie de la même institution.

${ }^{2}$ Conseiller d'orientation. Master en psychologie en tant que science et profession (PUCCamp), spécialiste en éducation et psychopédagogie (PUCCamp), spécialiste en neuropsychologie (Hôpital Hospitalier Institut Albert Einstein), Diplômé en psychologie (Fondation Hermínio Ometto - FHO).
}

RC: 98977

Disponible en: https://www.nucleodoconhecimento.com.br/psychologie-fr/aspectshistoriques 
possibles sur la victime. Pour cela, une revue bibliographique de caractère qualitatif, descriptif et exploratoire a été réalisée, suscitant des réflexions sur le thème. On a vu que l'enfant et l'adolescent n'avaient pas toujours un soutien juridique, ayant subi plusieurs violences sans la punition due à leurs agresseurs. Au fil du temps, des lois ont été instituées et, au Brésil, le Statut des enfants et des adolescents (ECA) a marqué une période de détermination des droits de l'enfance et de l'adolescence, établissant des mécanismes de protection, y compris contre la violence telle que les abus sexuels. Enfin, il a été considéré dans cette étude qu'il y a un manque d'information de la population sur le sujet, ce qui corrobore la reproduction de l'infraction susmentionnée. II a été observé que, compte tenu des dommages causés à la santé biopsychosociale de la victime et de sa famille, des études et des projets de prévention ont été réalisés, ainsi que mis en place différentes formes de signalement de ce crime. Il a été conclu qu'il est nécessaire d'élaborer des stratégies pour préparer les familles, les professionnels et les institutions chargées de garantir les droits des enfants et des adolescents afin qu'ils puissent contribuer à renforcer la lutte contre les abus sexuels.

Mots-clés : Abus sexuel, Agression sexuelle d'enfants, Enfant et adolescent.

\section{INTRODUCTION}

Lorsqu'il s'agit d'un sujet présent dans différentes parties du monde et à diverses périodes historiques, les abus sexuels restent aujourd'hui et constituent un sujet important à aborder, car cette violence a été continuellement reproduite. Waiselfisz (2012) montre que, selon les registres du Système d'information sur les maladies à déclaration obligatoire (SINAN), en 2011, 10425 enfants victimes d'abus sexuels ont été traités au Brésil. En 2018, le Brésil a enregistré au moins 32000 cas d'abus sexuels contre des enfants et des adolescents en 2018, le taux de notifications le plus élevé jamais enregistré par le ministère de la Santé. Depuis 2011, les agents de santé ont l'obligation de calculer les soins. Depuis lors, les chiffres augmentent d'année en année et totalisent 177300 notifications à l'échelle nationale[3].

RC: 98977

Disponible en: https://www.nucleodoconhecimento.com.br/psychologie-fr/aspectshistoriques 
Cependant, comme l'indiquent Aded et al. (2006), on croit que les cas identifiés ou signalés ne renseignent pas sur la réalité du nombre de cas. L'une des raisons est que, dans la plupart des cas, l'agresseur est très proche ou fait partie de la famille de la victime; un autre facteur est l'absence d'identification des cas, ou même la croyance qu'il n'y a aucun moyen de prouver l'acte d'abus, ne comprenant comme preuve concrète que l'examen médical. Ainsi, on estime que le nombre de cas est beaucoup plus élevé que prévu.

Sur la base des facteurs décrits, cette recherche présente la question directrice suivante : qu'est-ce que l'abus sexuel et quels sont ses impacts sur le développement de l'enfant? Cet article visait à passer en revue les aspects historiques et juridiques de l'abus sexuel, en explorant la complexité de ses définitions et en présentant les effets possibles sur la victime.

II s'agit d'une revue bibliographique à caractère qualitatif, descriptif et exploratoire, pour laquelle des livres ont été utilisés comme source de données, en plus des articles scientifiques qui ont été trouvés sur le site de recherche Google. Certains sites qui présentent des données importantes sur le sujet ont également été utilisés dans cette recherche.

Considérant que l'abus sexuel est une violence qui se produit d'une manière qui entrave habituellement son identification, en plus de provoquer de nombreux doutes sur la façon d'y faire face, il est nécessaire de développer davantage de recherches afin que de plus en plus de connaissances sur les mécanismes qui impliquent ce crime et, par conséquent, plus de stratégies de combat soient établies. Ainsi, cette recherche montre sa pertinence sociale et scientifique, contribuant à la clarification sur le sujet et à d'autres recherches à effectuer à partir de celui-ci.

RC: 98977

Disponible en: https://www.nucleodoconhecimento.com.br/psychologie-fr/aspectshistoriques 


\title{
2. ABUS SEXUELS: DÉFINITIONS, HISTOIRES, LOIS
}

L'abus sexuel est un phénomène social qui nécessite une attention particulière parce qu'il s'agit d'une question complexe, qui implique différents problèmes et génère des souffrances pour de nombreux enfants, adolescents et familles, ce qui est l'un des nombreux types de violence. Compte tenu des différentes définitions de l'abus sexuel, on peut souligner celle donnée par le Ministère de la santé (BRASIL, 2002, p.13) :

\begin{abstract}
Abuso sexual - consiste em todo ato ou jogo sexual, relação heterossexual ou homossexual cujo agressor está em estágio de desenvolvimento psicossexual mais adiantado que a criança ou o adolescente. Tem por intenção estimulá-la sexualmente ou utilizá-la para obter satisfação sexual. Apresenta-se sobre a forma de práticas eróticas e sexuais impostas à criança ou ao adolescente pela violência física, ameaças ou indução de sua vontade. Esse fenômeno violento pode variar desde atos em que não se produz o contato sexual (voyerismo, exibicionismo, produção de fotos), até diferentes tipos de ações que incluem contato sexual sem ou com penetração. Engloba ainda a situação de exploração sexual visando lucros como é o caso da prostituição e da pornografia.
\end{abstract}

Ainsi, étant considéré comme un acte de violence, l'abus sexuel peut être commis de différentes manières, de celles dans lesquelles il n'y a pas de contact physique entre l'agresseur et l'enfant ou l'adolescent, comme le voyeurisme dans lequel l'observation de la nudité a lieu. la satisfaction et l'exhibitionnisme, dans lesquels l'agresseur exhibe ses propres organes sexuels. Elle peut également se produire en montrant à la victime des vidéos et des magazines pornographiques, éveillant sa sexualité de manière précoce et déformée ; donner de l'argent, des bonbons ou des cadeaux à l'enfant ou à l'adolescent pour permettre à l'agresseur d'abuser de son corps ; la violer avec le sexe oral, qui est aussi un moyen de transmettre des maladies sexuellement transmissibles (MST) ; ou même avoir des relations sexuelles vaginales et anales. Cela peut se produire même sans que la victime s'en rende compte, ou au point de causer des blessures physiques et des maladies psychologiques. De plus, de tels abus ne sont pas seulement commis par des

RC: 98977

Disponible en: https://www.nucleodoconhecimento.com.br/psychologie-fr/aspectshistoriques 
hommes adultes sur des enfants : ils peuvent être perpétrés par des adolescents, des femmes, des personnes âgées et par différentes personnes dans différentes circonstances. Cette variation dans les façons dont les abus sexuels peuvent se produire peut rendre difficile l'identification de certains types qui ne sont souvent pas reconnus par les adultes en tant que tels (BRASIL, 2002).

En outre, comme l'a ponctué le Ministère de la santé (BRASIL, 2002), l'abus sexuel englobe l'exploitation sexuelle, c'est-à-dire lorsqu'il s'agit d'argent, de cadeaux ou de quelque chose du genre, dans une commercialisation du corps de l'enfant et/ou de l'adolescent, comme dans les exemples mentionnés, qui sont la prostitution et la pornographie. Cependant, comme le soulignent Vega et Paludo (2015, p.48), « définir l'exploitation sexuelle a été une tâche ardue, y compris pour le domaine universitaire ". Faleiros et Campos (2000), dans une analyse du vocabulaire du thème, ont identifié une inexactitude terminologique. Les auteurs donnent un exemple : l'abus sexuel a été établi comme un crime sexuel, la victimisation sexuelle, l'abus sexuel, l'agression sexuelle, la violence sexuelle, l'exploitation sexuelle, l'abus sexuel, l'outrage sexuel et les blessures sexuelles. Afin de nommer la violence intrafamiliale, les termes suivants ont été trouvés: inceste, abus sexuel incestueux, violence sexuelle domestique, abus sexuel domestique.

O uso sexual de menores de idade com fins lucrativos é designado ora como prostituição infanto-juvenil, ora como abuso sexual, ora como exploração sexual comercial. Por outro lado, um mesmo termo pode designar distintas realidades, como, por exemplo, o termo exploração sexual é utilizado pela OMS para designar situações de abuso sexual intra e extrafamiliar e prostituição, enquanto muitos autores o utilizam referindo-se apenas à exploração sexual comercial (FALEIROS; CAMPOS, 2000, p.4).

Ainsi, il existe également des divergences entre les auteurs en ce qui concerne le vocabulaire approprié pour chaque situation. Néanmoins, pour Faleiros et Campos (2000), l'utilisation de termes différents comme synonymes et comme s'ils étaient équivalents au même concept, ce n'est pas seulement de la terminologie, mais une question de portée épistémologique, c'est-à-dire dénonce le manque de

RC: 98977

Disponible en: https://www.nucleodoconhecimento.com.br/psychologie-fr/aspectshistoriques 
conceptualisation du problème. Cette incertitude théorique et conceptuelle est due à la complexité et à la diversité, ainsi qu'au fait que l'annulation, la prise de conscience et la gestion de ce problème sont encore récentes, ainsi qu'au souci de l'enfance, comme on le verra ci-dessous.

En plus de se produire de différentes manières, les abus sexuels sur enfants proviennent de différents endroits de la planète et sont décrits depuis l'Antiquité, comme le montrent Aded et al. (2006). Cependant, les progrès dans la prévention et l'atténuation des conséquences ont été peu importants. Ce qui peut rendre difficile de faire face au problème, c'est le fait de traverser des complexités culturelles, religieuses, juridiques et professionnelles du monde entier.

Oliveira (2006) souligne que tout au long de l'histoire, l'une des caractéristiques qui ont marqué la culture humaine a été la négligence contre les enfants et les adolescents, car il n'y avait aucune idée de la fragilité comme caractéristique de l'enfance, et cette question a été peu discutée, tout comme il n'y avait pas de politiques de protection.

L'auteur présente plusieurs points de l'histoire qui illustrent cette négligence, indiquant que dans de nombreux peuples de l'Antiquité, l'abus sexuel d'enfants était considéré comme normal et, de plus, beaucoup de ces peuples ne faisaient pas de distinction entre l'enfant et l'adulte, comme à Sparte, par exemple. II indique également qu'en Occident, au Moyen Âge, cette distinction n'a pas non plus été faite et que l'enfant est resté exposé à différents types de punitions; « [...] dès qu'elle n'avait pas besoin de soins maternels de base, elle était considérée comme une adulte et grandissait donc »(OLIVEIRA, 2006, p. 11). Ainsi, le très jeune enfant n'était pas considéré comme un être humain et le taux de mortalité était élevé, un fait qui a été compris comme un phénomène commun.

Azambuja (2006, p.3) présente que ce n'est qu'à la fin du 17ème siècle que l'enfant a commencé à se distinguer de la personne adulte. Jusqu'à cette époque, les écoles

RC: 98977

Disponible en: https://www.nucleodoconhecimento.com.br/psychologie-fr/aspectshistoriques 
n'étaient pas organisées par âge, étant fréquentées par des enfants et des adultes au même endroit. Pour l'auteur, « [...] avec l'émergence de la compréhension que l'enfance est une phase distincte de la vie adulte, les châtiments, les châtiments corporels, les coups à coups de fouet, de bâtons et de fers sont utilisés comme instruments nécessaires à l'éducation " (AZAMBUJA, 2006, p.3). En 1780, en Angleterre, les parents pouvaient condamner les enfants à la pendaison, sous plus de deux cents types criminels. À New York, en 1871, une jeune fille a subi de graves mauvais traitements et, comme il n'y avait pas de place pour une telle plainte, le cas de la fille Mary Ellen a finalement été renvoyé à la Società per la prevenzione della crudeltà verso gli animali, afin qu'il puisse faire l'objet d'une enquête par le tribunal. L'affaire, qui a provoqué beaucoup d'indignation de la population, a incité à la fondation d'une société axée sur la protection de l'enfant.

Selon Azambuja (2006), en ce qui concerne le Brésil, les premiers navires lancés en mer par le Portugal étaient composés d'hommes et d'enfants orphelins du roi, qui ont fourni des services pendant le voyage et ont été victimes d'abus sexuels de la part des marins. Elle dit que si des tempêtes se produisaient, les enfants étaient les premières « cargaisons » à être jetées par-dessus bord.

Guerra et al. (2013) soulignent que jusqu'au début du 20e siècle, l'État ne se préoccupait pas de développer des politiques sociales destinées aux enfants et aux adolescents. Ainsi, c'est l'Église catholique qui a développé des actions importantes pour prendre soin des enfants et des adolescents dits « abandonnés ». Les Saintes Maisons de la Miséricorde ne s'occupaient pas seulement des malades et des malades, mais aussi des enfants dont les droits avaient été violés. Ainsi, dans une tentative de réduire le nombre de bébés conçus hors mariage et qui ont été abandonnés même dans la rue, en raison des pressions sociales de l'époque, l'Église catholique a créé la Roue des Exposés. II s'agissait d'un dispositif cylindrique dans lequel l'enfant était placé et laissé, afin d'assurer l'anonymat de ceux qui ont commis l'abandon.

RC: 98977

Disponible en: https://www.nucleodoconhecimento.com.br/psychologie-fr/aspectshistoriques 
À la fin de la Première Guerre mondiale, des organisations internationales nouvellement créées ont commencé à articuler des codes des droits de l'homme. De nouveaux organes ont commencé à prêter attention aux droits de l'enfant en particulier. L'Agence internationale du travail nouvellement créée (aujourd'hui l'Organisation) a rendu ses conventions pour garantir les droits des enfants qui travaillent, ainsi qu'en 1919, à la Convention sur le travail de nuit des adolescents (industrie); également en 1921, la Convention sur l'âge minimum (agriculture). Cependant, la plupart des lois produites entre les deux guerres mondiales n'expliquaient pas la spécificité du droit des enfants et des adolescents par opposition aux droits directs des adultes (UNICEF, 2009).

La première définition des droits formels de l'enfant, qui a été créée par les organisations internationales naissantes, provient du travail d'Eglantyne Jebb qui a fondé Save the Childrenen Angleterre en 1919 et a créé à Genève l'année suivante, I'Union internationale Save the Children, pour collecter des fonds dansl'aide d'urgence aux enfants souffrant de la Première Guerre mondiale. Jebb, en 1923, a déclaré sa position sur les droits de l'enfant : « [...] Je crois que nous devons revendiquer certains droits pour l'enfant et travailler pour qu'ils soient reconnus internationalement " (UNICEF, 2009, p.4). Ainsi, I'Union internationale Save the Children a créé la proposition préliminaire d'une déclaration revendiquant des droits spécifiques à la population enfantine et a fait adopter par la Société des Nations dans la Déclaration de Genève des droits de l'enfant le 26 septembre 1924.

Ainsi, la Déclaration de Genève a examiné cinq principes fondamentaux : le droit aux conditions du développement matériel et spirituel ; le droit de priorité dans les situations à risque; le droit d'aider en cas de faim, d'invalidité, de maladie, de délinquance ou d'orphelinat; le droit à une formation qui guide l'enfant vers la vie en société; et le droit à la protection contre l'exploitation (UNICEF, 2009).

Selon le Fonds des Nations Unies pour l'enfance (UNICEF, 2009), la Seconde Guerre mondiale a corroboré la création des Nations Unies (ONU). La fusion entre

RC: 98977

Disponible en: https://www.nucleodoconhecimento.com.br/psychologie-fr/aspectshistoriques 
l'Association internationale pour le bien-être del'enfant, établie à Bruxelles et l'Union internationale Save the Children, a abouti à l'Union internationale pour la protection de l'enfance (International Union for Child Welfare - IUCW), qui a conduit les Nations Unies à approuver la Déclaration de Genève.

Le site Web de Portal Brasil[4] montre qu'en 1927, le pays a promulgué le premier Code des mineurs. À l'époque, des cas très médiatisés de grandes répercussions publiques ont incité le président de l'époque, Washington Luís, à déterminer que 18 ans devraient être l'âge minimum pour les délinquants en prison. Ce qui est arrivé au garçon Bernardino, âgé de 12 ans, était l'un de ces cas. En 1926, le garçon, qui était cireur de chaussures, s'est fâché contre un client en raison de son refus de payer pour le service fourni dans les rues de Rio de Janeiro. L'enfant aurait jeté de la peinture sur ce type, alors elle s'est retrouvée en prison pendant quatre semaines. Bernardino a été violée dans la cellule par 20 adultes, ce qui a provoqué l'indignation des médecins lorsque le garçon est allé à Santa Casa.

Ainsi, la propagation de l'affaire a provoqué une forte controverse, initiant un débat public qui a atteint les rouages du Congrès, ainsi que le palais Catete, où il était la place du gouvernement fédéral. Le président a donc choisi le 12 octobre (Journée de l'enfance) pour signer le Code des mineurs, qui était la première législation du pays pour l'enfance et l'adolescence en particulier. Un article de ce code interdisait la Roue des Exposés. « Avec le code, la mère devrait enregistrer l'enfant et ainsi l'a livrer à un orphelinat » (BRASIL, s/d).

Le site de la Déclaration universelle des droits de l'homme[5] (DUDH) montre que le 10 décembre 1948, à Paris, une telle déclaration a été proclamée par l'Assemblée générale des nations, par la résolution 217 A (III). C'est un document qui a marqué I'histoire des droits de l'homme. La DUDH a été développée par différents représentants de divers milieux juridiques et culturels du monde entier, et est alors une norme commune afin qu'elle atteigne tous les peuples et toutes les nations. Cette déclaration vient avec l'idée que l'homme a droit à une vie digne : «

$\mathrm{RC}: 98977$

Disponible en: https://www.nucleodoconhecimento.com.br/psychologie-fr/aspectshistoriques 
Considérant que la reconnaissance de la dignité inhérente à tous les membres de la famille humaine et de leurs droits égaux et inaliénables est le fondement de la liberté, de la justice et de la paix dans le monde [...] » (DUDH, 1948).

À cette occasion, l'ONU s'est montrée plus déterminée à discuter des principes de la DUDH, mais l'UIW elle-même a défendu l'idée d'un nouveau document, spécifique aux droits de l'enfant, mettant à jour la déclaration précédente et réitérant le principe selon lequel l'enfant devrait recevoir ce que l'humanité a de mieux à offrir. La Déclaration des droits de l'enfant n'a été adoptée par l'ONU que le 20 novembre 1959. L'appui de l'Assemblée générale est important, car il souligne la nécessité d'examiner séparément les droits de l'enfant. La Déclaration des droits de l'enfant a mis l'accent sur le bien-être émotionnel de l'enfant et a garanti son droit d'être prioritaire dans l'obtention d'une protection d'urgence. "Malgré ces changements, le document de 1959 a maintenu une approche de recherche de soins, visant à sauver, garder et protéger l'enfant, accordant peu d'importance à l'augmentation de son pouvoir » (UNICEF, 2009, p.5).

Inoue et Ristum (2008) indiquent que la violence a été la principale cause de décès, principalement chez les jeunes et les enfants. Aux États-Unis, ce n'est qu'en 1960 que la violence contre les enfants a été considérée comme un problème médical et social. " [...] en particulier, les pédiatres ont commencé à traiter le thème montrant les séquelles de la maltraitance et les divergences entre les preuves radiologiques sur les blessures et les explications des agresseurs " (MINAYO, 2002, p. 109). Bien que ce mouvement au sein de la médecine ait provoqué des critiques et des accusations de "médicalisation de la violence ", il a eu une grande influence sur la production théorique, déclenchant la recherche, ainsi que sur les mouvements en faveur de la défense des droits de l'enfant. Ainsi, le domaine médical a commencé une répulsion sociale sur les abus sexuels, qui a pris de la cohérence également des États-Unis, tout en renforçant le mouvement féministe de 1970.

RC: 98977

Disponible en: https://www.nucleodoconhecimento.com.br/psychologie-fr/aspectshistoriques 
L'année 1973 a été marquée par un événement qui a fait du 18 mai, un jour important dans le calendrier du ministère de la Santé, un mémorial jusqu'à nos jours. Le fait est qu'à cette date, Araceli Cabrera Crespo, neuf ans, a disparu de l'école où elle étudiait, n'étant plus vue vivante. Le site Web du Conseil national de la santé explique que «Araceli a été battue, violée, droguée et tuée dans une orgie de drogues et de sexe. Son corps, son visage était principalement défiguré par l'acide » (BRASIL, s/d). Six jours après l'incident, le corps a été retrouvé dans un terrain vague près du centre-ville de Vitória, Espírito Santo. Ce martyre ayant une grande importance, cette date est considérée et rappelée comme la Journée nationale de lutte contre les abus sexuels et l'exploitation sexuelle des enfants et des adolescents.

Entre les années 1960 et 1970, la mobilisation pour les droits des enfants et des adolescents a été faite par des organisations non gouvernementales (ONG), ce qui a incité à de grands progrès; ces organisations ont ainsi exhorté l'ONU à déclarer 1979 Année internationale de l'enfance, dans une proposition visant à attirer l'attention sur le problème de l'enfance. "Une fois cet accord établi, le Gouvernement polonais a soumis à la Commission des droits de l'homme des Nations Unies un texte préliminaire de la Convention relative aux droits de l'enfant » (UNICEF, 2009, p. 5). Ainsi, il a été démontré que l'achèvement de ce document nécessiterait une meilleure préparation et plus de temps. Ainsi, le processus a duré une décennie, considérant que la rédaction d'un traité impliquant plusieurs domaines d'interprétation culturelle et sociale est un travail qui nécessite de la prudence.

Minayo (2002) dit que dans les années 1980, dans le domaine de la santé publique mondiale, la violence se présente comme une question de fond. Les mouvements de soins spécialisés et de prévention découlent de la reconnaissance de la morbidité et de la mortalité par la violence, en tant que problème grave pour la santé dans ses aspects sociaux, ainsi que la cible des soins primaires, secondaires et tertiaires. Toujours en 1980, au Brésil, des propositions ont commencé à être pensées, au rythme du mouvement social qui corroborait la Constitution de 1988. Ce n'est que

$\mathrm{RC}: 98977$

Disponible en: https://www.nucleodoconhecimento.com.br/psychologie-fr/aspectshistoriques 
cette année que l'enfant est considéré comme un sujet de droits, grâce à la Constitution fédérale, et aura la Convention des Nations Unies, dans une nouvelle ère de défense des droits des enfants et des adolescents.

Trois ans avant cette constitution, le 4 juillet 1985, le Centre régional pour la prise en charge de la maltraitance des enfants (CRAMI) a été fondé par un groupe composé de différents professionnels appartenant à l'Université pontificale catholique de Campinas (PUC-Campinas), ayant impliqué des représentants de divers secteurs de la communauté, tels que l'Association du barreau brésilien (OAB) - Campinas, Tribunal pour enfants, entre autres. Selon le propre site Web de CRAMI[6], « Préoccupé par les cas courants d'enfants arrivant dans les organismes publics, présentant des marques de coups, de brûlures, d'abrasions, d'hémorragies internes, ce groupe a fondé CRAMI ". Beaucoup de ces enfants sont morts, et les responsables l'ont justifié comme une chute ou un manque d'attention de l'enfant luimême. Au fil du temps, un protocole a été établi pour s'occuper des cas de violence familiale. À partir de la conclusion de l'affaire, des données ont été recueillies et le travailleur social a fourni une procédure de prise en charge dans les foyers des victimes, dans le but d'avoir un contact avec la situation réelle et, en fonction de la gravité de l'affaire, de renvoyer la victime devant le tribunal de l'enfance.

Le site Web de CRAMI informe également que «le travail développé par les professionnels hautement qualifiés de CRAMI a commencé à devenir une référence et a encouragé l'ouverture d'autres CRAMI dans l'État de São Paulo ». Par la détermination du Système unifié d'assistance sociale (SUAS), CRAMI a étendu en 2014 ses soins à toutes les personnes en situation de vulnérabilité et de risque social, ne se limitant plus à la prise en charge des enfants et des adolescents victimes de violence domestique. II s'agit d'une entité non gouvernementale à but non lucratif, qui bénéficie de l'aide de psychologues, de travailleurs sociaux et d'éducateurs sociaux, qui poursuit la mission, selon le site Web, de «Construire un avenir meilleur pour les enfants, les adolescents et les victimes vulnérables de violence domestique ».

$\mathrm{RC}: 98977$

Disponible en: https://www.nucleodoconhecimento.com.br/psychologie-fr/aspectshistoriques 
Le 20 novembre 1989, l'Assemblée générale des Nations Unies a adopté la Convention relative aux droits de l'enfant, qui est entrée en vigueur le 2 septembre 1990. II s'agit du plus complet de tous les traités relatifs aux droits de l'homme et des instruments juridiques relatifs à la protection des droits de l'enfant. Bien qu'il existe des dispositions pour la défense des droits de l'enfant dans les équipements internationaux relatifs aux droits de l'homme, la Convention est la première à couvrir et à articuler tous les aspects pertinents des droits importants pour l'enfant, qu'ils soient économiques, culturels, politiques et sociaux. Pourtant, c'était le premier équipement international qui reconnaissait explicitement que l'enfant est un acteur social possédant ses propres droits (UNICEF, 2009).

Selon I'UNICEF (2009, p. 2), « En application des dispositions du traité, les États parties sont tenus par la loi de respecter les droits de tous les enfants ». La Convention prévoit 54 articles et repose sur quatre principes fondamentaux : l'intérêt supérieur de l'enfant; la non-discrimination; le respect des opinions de l'enfant; et le droit à la survie et au développement. L'objectif général qu'il a et l'importance qu'il accorde à la représentation qui favorise l'enfant déterminent la pertinence incessante de toutes les actions visant à satisfaire aux droits de l'enfant. "La Convention réaffirme et enrichit les droits de l'homme de manière significative » (UNICEF, 2009, p. 2). II les réitère en appliquant directement à l'enfant bon nombre des principes essentiels des documents internationaux relatifs aux droits de l'homme qui préexistaient, tels que la non-discrimination et l'universalité; les renforce en mettant en place et en élargissant les dispositifs inclus dans d'autres équipements relatifs aux droits de l'homme, en clarifiant les responsabilités des États parties à l'égard de l'enfant. II intègre les droits de l'enfant qui n'ont pas été largement incorporés. II souligne que la responsabilité de la réalisation des droits de l'enfant devrait être attribuée à ceux qui ont des devoirs à son égard, tels que la famille et les personnes responsables de l'enfant et les États parties.

La convention a un sens qui va au-delà de ses implications juridiques. L'instrument a également contribué à une transformation des attitudes envers l'enfance. En outre, la

$\mathrm{RC}: 98977$

Disponible en: https://www.nucleodoconhecimento.com.br/psychologie-fr/aspectshistoriques 
Convention a déterminé les conditions de l'enfance, en fixant les normes minimales en matière de soins, de traitement, de survie, de protection, de participation et de développement, qui sont les droits de toutes les personnes dont l'âge est âgé de moins de 18 ans. Ses articles réaffirment qu'il est nécessaire de protéger l'enfance en tant que période différente de l'âge adulte et d'établir un moment pendant lequel l'enfant grandit, apprend, joue et se développe, afin que les droits de l'enfant soient pleinement réalisés (UNICEF, 2009).

Comme le montre Lemos (2008), inaugurant une nouvelle perspective des droits et des devoirs fondée sur la doctrine de la protection intégrale, le Statut des enfants et des adolescents (ECA) a été promulgué en 1990, en tant que loi fédérale $n^{\circ} 8$ 069/90, garantissant à l'enfant et à l'adolescent le respect en tant que personnes en particulier le développement:

Art. 3o A criança e o adolescente gozam de todos os direitos fundamentais inerentes à pessoa humana, sem prejuízo da proteção integral de que trata esta Lei, assegurando-se Ihes, por lei ou por outros meios, todas as oportunidades e facilidades, a fim de thes facultar o desenvolvimento físico, mental, moral, espiritual e social, em condições de liberdade e de dignidade (BRASIL, 1990).

Cette loi met en place un nouveau schéma, établissant des pratiques différentes dans certains aspects de celles prévues par le Code des mineurs de 1927 et 1979. « $\mathrm{Si}$, dans le Code des mineurs, l'objet était le plus petit ; dans le Statut de l'enfant et de l'adolescent, l'objet est l'enfant et l'adolescent en tant que sujets de droits " (LEMOS, 2008, p.98). De cette façon, une nouvelle identité sociale appelée enfants et adolescents est inaugurée. Ce ne sont pas seulement les pratiques qui ont changé, et l'objet en est un autre.

L'ECA vient avec la proposition de construire un modèle de protection intégrale pour les enfants et les adolescents, ne se limitant pas uniquement aux soins après la violation des droits, mais avant que la violation ne se produise, comme le prévoit son article oc de menace ou de violation des droits des enfants et des adolescents »

RC: 98977

Disponible en: https://www.nucleodoconhecimento.com.br/psychologie-fr/aspectshistoriques 
(BRASIL, 1990). Cette loi fédérale assure, entre autres aspects, la protection des enfants contre tout type de violence, y compris sexuelle.

On sait que, dans la plupart des cas, l'agresseur a une proximité avec la famille. Comme le montrent Santos, Costa et Granjeiro (2009), la plupart des cas d'abus sexuels se produisent au sein de la famille et sont pratiqués d'une part par les parents biologiques des victimes et, d'autre part, par les beaux-pères. C'est un exemple de violence intrafamiliale qui, selon Araújo (2002), est la violence qui se produit dans la famille, impliquant des personnes qui vivent ou non au même endroit, mais qui ne se limite pas à la famille, mais implique des sujets de convivialité commune dans le même espace domestique, qu'ils aient ou non des liens de parenté. Pour ces cas, la Cour a des spécificités et l'une d'entre elles est mentionnée à l'art. 101:

$\S 2^{\circ}$ Sem prejuízo da tomada de medidas emergenciais para proteção de vítimas de violência ou abuso sexual e das providências a que alude 0 art. 130 desta Lei, o afastamento da criança ou adolescente do convívio familiar é de competência exclusiva da autoridade judiciária e importará na deflagração, a pedido do Ministério Público ou de quem tenha legítimo interesse, de procedimento judicial contencioso, no qual se garanta aos pais ou ao responsável legal o exercício do contraditório e da ampla defesa (BRASIL, 1990).

Ces lois visant à protéger les enfants et les adolescents contre les abus sexuels sont extrêmement nécessaires, car elles traitent de quelque chose de grave et peuvent causer de nombreux préjudices à la victime, comme on le soulignera ci-dessous.

\subsection{DOMMAGES CAUSÉS À LA VICTIME ET ACTIONS POSSIBLES}

Parmi les conséquences que les abus sexuels peuvent causer aux enfants et aux adolescents, on peut souligner: les déficiences cognitives, comportementales, émotionnelles et sociales; isolement social, peur exagérée, difficultés d'adaptation, idées homicides et suicidaires, apprentissage du déficit linguistique, perte d'intérêt pour les jeux et les études, évasions de la maison, automutilation, isolement social,

RC: 98977

Disponible en: https://www.nucleodoconhecimento.com.br/psychologie-fr/aspectshistoriques 
agressivité et autres conséquences pouvant sérieusement compromettre la vie de la victime (SERAFIM et al., 2011).

Prado et Carneiro (2005) discutent, du point de vue de la psychanalyse, du traumatisme que, selon eux, le terme mettrait l'accent sur les dommages générés dans la capacité de symboliser et de transformer, ainsi que la corroboration des zones psychiques mortes, qui interféreront dans les générations futures, atteignant leurs choix d'amour et leur perspective de réalisation de leur sexualité.Les auteurs discutent de deux types de traumatismes, proposant de les appeler actifs et cumulatifs, et le premier reste dans la psyché de la personne à plusieurs reprises, en particulier lorsqu'il s'agit d'abus sexuels sur enfants, et peut avoir les diverses manifestations, telles que la promiscuité, les performances, les conditions psychopathiques, les dépressions, les conditions psychosomatiques, etc.

Le traumatisme cumulatif fait référence à la tension récurrente de nonaccomplissement du rôle de la mère en tant que bouclier protecteur, ce qui laisse dans la phase de développement que le bébé a besoin de la mère exerçant son rôle; ces échecs maternels peuvent être corrigés en fonction du processus de maturation. Quant au traumatisme actif, « de la même manière que le traumatisme cumulatif, il est perfide, mais au lieu d'agir et de s'installer tout au long de l'enfance et de l'adolescence, il le fait tout au long de la vie, et au-delà, puisqu'il est réédité à travers les générations » (PRADO; CARNEIRO, 2005, p. 31). Par conséquent, le traumatisme peut interférer et causer de la souffrance tout au long de la vie de la victime, apportant plusieurs possibilités d'effets graves à long terme.

La relation de l'enfant avec des adultes violents, entraînant la rupture de la confiance et de l'insécurité, favorise la victime à ne pas croire en la fiabilité et la sécurité des personnes en général, ce qui peut se refléter sur leurs relations futures. Ainsi, dès l'adolescence, il y a des cas de victimes qui, désespérées d'obtenir de l'affection, finissent par devenir promiscuités; d'autre part, les autres se rendent rarement

RC: 98977

Disponible en: https://www.nucleodoconhecimento.com.br/psychologie-fr/aspectshistoriques 
disponibles pour les relations; les deux cas évoluent vers l'isolement et la dépression (PRADO; CARNEIRO, 2005).

Padilha (2002) parle de la particularité de l'abus sexuel, soulignant qu'il n'y a souvent aucune preuve physique de sa consommation, puis l'agresseur soutient qu'il n'a pas forcé l'enfant à faire quoi que ce soit. "Considérer cette caractéristique du phénomène semble être fondamental dans la planification des interventions pour la prévention ou le traitement des situations d'abus sexuels, intrafamiliaux ou extrafamiliaux »(PADILHA, 2002, p. 210). Ainsi, la prévention d'une telle violence nécessite une connaissance des mouvements qui imprègnent les agressions.

Comme déjà mentionné, le Ministère de la santé souligne que les abus sexuels « se présentent sous la forme de pratiques érotiques et sexuelles imposées aux enfants ou aux adolescents pour la violence physique, les menaces [...] 》 mais complètes : « [...] ou l'induction de leur volonté » (BRASIL, 2002, p.13). Ainsi, en fait, la plupart des cas d'abus sexuels se produisent sous la menace de l'agresseur, à l'ombre de la violence, du chantage, etc., cependant, induire la volonté de la victime constitue également une agression.

II existe encore actuellement un concept erroné qui considère que si l'enfant et surtout l'adolescent sont de connivence avec l'acte sexuel, la situation ne constituerait pas un abus. Cependant, la loi brésilienne ne laisse aucun doute sur le fait que les enfants et les adolescents sont vulnérables car, comme cela a déjà été apporté à cette recherche, si la personne est à un stade de développement psychosexuel plus tôt que la victime, il s'agira d'abus sexuels (BRASIL, 2002).

Par conséquent, même si l'enfant ou l'adolescent consent à l'acte sexuel, cela est clairement répréhensible par la législation brésilienne. Abordant les crimes sexuels contre les personnes vulnérables, l'article 217-A du Code pénal indique clairement que « Avoir une conjonction charnelle ou pratiquer un autre acte libidineux de moins

RC: 98977

Disponible en: https://www.nucleodoconhecimento.com.br/psychologie-fr/aspectshistoriques 
de 14 (quatorze) ans : Stylo - emprisonnement, de 8 (huit) à 15 (quinze) ans » (HARMS, 2016, p.577).

De plus, le 6 novembre 2017, la Cour supérieure de justice (STJ) a publié le résumé 593, qui détermine avec insistance qu'il n'est pas pertinent pour la caractérisation du crime le consentement ou non de la victime :

O crime de estupro de vulnerável se configura com a conjunção carnal ou prática de ato libidinoso com menor de 14 anos, sendo irrelevante eventual consentimento da vítima para a prática do ato, sua experiência sexual anterior ou existência de relacionamento amoroso com o agente. (Súmula 593, TERCEIRA SEÇÃO, julgado em 25/10/2017, DJe 06/11/2017) (BRASIL, 2017).

Ainsi, la législation brésilienne indique encore plus clairement que, quelles que soient les circonstances d'acceptation ou non de l'enfant ou de l'adolescent, les facteurs mentionnés au sujet des abus sexuels s'appliquent et sont constitués en tant que crimes.

De plus, le fait que la victime consente d'une manière ou d'une autre peut provoquer une augmentation du sentiment de culpabilité et aggraver les dommages émotionnels, en plus de corroborer son silence sur la violence subie et, dans de nombreux cas, de favoriser l'enfant ou l'adolescent à continuer d'être abusé sexuellement. Pour Padilha (2002), la durée de l'abus peut consister en un épisode isolé ou de routine de plusieurs années sans divulgation à des tiers. L'agression peut se faire avec des victimes de tout âge, des bébés ou des très jeunes enfants, à l'adolescence.

En outre, les hypothèses sur le comportement de collusion avec une telle violence sont établies en fonction du comportement de la mère, du père ou du beau-père, ou de l'enfant, provenant de l'histoire de la vie, qui répète parfois les schémas des générations passées. L'absence d'un répertoire d'autoprotection peut être due à des modèles inadéquats de parents, qui n'ont peut-être pas eu un modèle adéquat de leurs parents, et ainsi de suite. «Ce mécanisme de répétition intergénérationnelle

RC: 98977

Disponible en: https://www.nucleodoconhecimento.com.br/psychologie-fr/aspectshistoriques 
des comportements d'exposition au risque expliquerait pourquoi les mères, les pères ou les beaux-pères deviennent "aveugles " par rapport aux abus sexuels [...] " (PADILHA, 2002, p.211). C'est-à-dire qu'ils ne peuvent pas identifier les preuves de violence.

En outre, le stade de développement dans lequel se trouve la victime est instrait à la fois dans le processus de découverte et dans le procès du suspect: « La maltraitance des enfants est un crime qui ne peut souvent être prouvé que par le témoignage d'enfants d'âge préscolaire. Si le témoignage d'un enfant est incorrect, un adulte innocent peut être puni injustement » (PAPALIA; OLDS; FELDMAN, 2006, p. 301). Comme les auteurs le soulignent à juste titre, le jeune enfant peut finir par " se souvenir " d'événements qui ne se sont pas produits; ils ont tendance à être suggestifs et leur discours peut être induit par une autre personne, et il est difficile de discriminer le mensonge du discours d'un enfant qui est interrogé par quelqu'un qui pose des questions avec des détails biaisés. Cela peut se produire principalement chez les enfants de moins de quatre ans, car à ce stade, les enfants sont plus susceptibles de faire des erreurs dans la mémorisation des détails d'un événement qui varie avec la répétition. Néanmoins, Habigzang, Ramos et Ekoller (2011) qualifient le moment de révélation d'abus sexuel de crucial dans la vie de la victime, car il peut provoquer une revictimisation si les adultes ne croient pas en la parole de l'enfant et ne prennent pas les mesures de protection appropriées, qui devraient avoir un réseau de soutien affectif et social, composé de systèmes ou de personnes importantes pour la victime.

Nos casos de abuso sexual contra crianças e adolescentes compreende-se como constituinte da rede: família, escola, comunidade, Conselho Tutelar, Delegacia, Conselho de Direitos da Criança, Ministério Público e Juizado da Infância e Adolescência, abrigos, serviços de saúde (postos de saúde e hospitais) e assistência social (Centro de Referência da Assistência Social e Centro de Referência Especializado da Assistência Social) (HABIGZANG; RAMOS; KOLLER, 2011, p. 468).

RC: 98977

Disponible en: https://www.nucleodoconhecimento.com.br/psychologie-fr/aspectshistoriques 
Ainsi, toutes ces institutions ont la responsabilité de protéger la victime, en s'efforçant de planifier et de faire des interventions qui minimisent efficacement les effets de la violence subie par les enfants ou les adolescents (HABIGZANG; RAMOS; KOLLER, 2011). L'école se distingue par le fait qu'elle est un environnement présent dans des phases très importantes de la vie des enfants et des adolescents dans son développement.

Toujours en clarifiant l'implication dans le développement de la victime d'abus sexuels, Papalia; Olds et Feldman (2006) montrent les citations les plus courantes selon l'âge : à l'école maternelle, l'anxiété, les cauchemars et les comportements sexuels inappropriés se démarquent; chez les enfants d'âge scolaire, des comportements agressifs, des problèmes scolaires, la peur, l'hyperactivité et même la maladie mentale sont observés; enfin, les adolescents présentent généralement une dépression, des plaintes physiques, la fuite, des actions illégales, des comportements autodestructeurs ou suicidaires et la toxicomanie.

Santos; Costa et Granjeiro (2009) soulignent que la plupart des cas d'abus sexuels se produisent au sein de la famille et que les cas les plus courants d'abus sexuels sont pratiqués par les parents biologiques des victimes et, deuxièmement, par les beaux-pères. C'est un type de violence intrafamiliale qui, selon Araújo (2002), se produit dans la famille impliquant des personnes qui résident ou non au même endroit, mais qui n'est pas limitée à la famille, mais implique des sujets de convivialité commune dans le même espace domestique, qu'elles aient ou non des liens de parenté. Ainsi, on peut affirmer qu'il existe un risque que les gens idéalisent un certain stéréotype pour de tels agresseurs, ne payant pas pour voir que, même s'ils sont des travailleurs, des "parents de famille ", des contribuables, ont une bonne vie sociale, sont des gens riches, gentils et éduqués ou qui apparemment éduquent et prennent bien soin de leurs enfants, cela ne signifie pas que ces personnes ne peuvent pas commettre d'abus sexuels, même s'ils ont une conduite louable envers la société.

RC: 98977

Disponible en: https://www.nucleodoconhecimento.com.br/psychologie-fr/aspectshistoriques 
« La plupart des pédophiles n'ont pas de casier judiciaire et les gens dans leur vie les décrivent comme un travailleur, religieux et un soignant zélé de leur famille » (COHEN; MANNARINO, 2000a; apud HABIGZANG et al., 2005, p. 346).

En outre, les agresseurs avec des stéréotypes considérés socialement comme de bons citoyens peuvent provoquer l'accommodement des gens pour ne pas se soucier de la prévention avec les enfants et les adolescents concernant les abus sexuels, car cela peut également semer la confusion dans le processus de suspicion et d'identification de cette violence. De plus, selon la relation que l'agresseur a avec la famille, de nombreux cas ne sont pas signalés. Par exemple, une mère peut ne pas dénoncer son conjoint parce qu'elle lui fait confiance, ne pas donner de crédit à la plainte de l'enfant ou de l'adolescent parce qu'elle ne veut pas s'éloigner du conjoint ou parce qu'elle dépend de l'agresseur pour soutenir la famille, entre autres situations. Williams (2002) cite un autre aspect important, à connaître le fait que la violence sexuelle est encore actuellement considérée comme taboue et que souvent la victime elle-même est stigmatisée et a souvent un sentiment de culpabilité ou de honte; c'est, entre autres, l'une des raisons de la non-déclaration par des personnes qui font ou ne font pas partie de la famille de la victime.

Cependant, Habigzang et al. (2005) présentent la cartographie des facteurs de risque d'abus sexuels intrafamiliaux qui ont été identifiés dans les procédures judiciaires du Bureau du Procureur général du Rio Grande do Sul - Brésil, en analysant 71 dossiers qui présentent le profil des agresseurs; l'une des caractéristiques frappantes était que la plupart des agresseurs étaient au chômage ou avec un éventuel travail. "Le chômage est un facteur de risque de violence intrafamiliale, car il peut générer du stress et des conflits entre les membres de la famille »(HABIGZANG et al., 2005, p. 345). En outre, le soignant finit par être responsable de l'enfant ou de l'adolescent la plupart du temps, ce qui offre plus de possibilités d'abus sexuels. D'autres caractéristiques trouvées chez la plupart des agresseurs de cette étude sont: faible niveau d'éducation, mariés ou vivants maritally, consommateurs d'alcool ou de drogues, entre autres choses. II est

RC: 98977

Disponible en: https://www.nucleodoconhecimento.com.br/psychologie-fr/aspectshistoriques 
important de noter que ces caractéristiques ont été trouvées dans un pourcentage des cas étudiés et, bien qu'elles soient assez courantes, de nombreux agresseurs ne présentent aucune de ces caractéristiques. Ainsi, il est important que les adultes soient attentifs et recherchent des informations qui augmentent leurs connaissances sur le sujet et soient ainsi en mesure de créer un réseau de protection pour les enfants et les adolescents, en étant capable non seulement d'identifier, mais aussi de prévenir, de prévenir les abus sexuels.

Pour Brino et Williams (2005), les abus pédosexuels peuvent être évités par des actions qui peuvent être divisées en trois niveaux de prévention. Le premier niveau est le niveau primaire, qui vise à éliminer ou à réduire les facteurs sociaux, environnementaux et culturels propices aux actes d'agression. Ainsi, des actions visant à former des professionnels et d'autres personnes directement impliquées avec l'enfant peuvent être encouragées, telles que les enseignants. En outre, des actions peuvent être encouragées auprès de la population à risque, en promouvant l'éducation des enfants sur les risques d'abus sexuels.

Santos et Ippolito (2009) soutiennent que l'éducation sexuelle est le meilleur moyen de prévenir, et que malgré l'appartenance à l'adulte responsable de l'éducation des enfants et des adolescents, la responsabilité de les protéger contre les abus sexuels, un programme de formation continue est capable de préparer les enfants et les adolescents à se défendre contre cette violence.

Un autre moyen de prévention mentionné par les auteurs est l'inclusion sociale de l'enfant considéré comme " différent ", ou rejeté par les groupes. "Après l'assujettissement de l'enfant aux abus sexuels subis à la maison ou dans le quartier ou son silence devant lui, il y a généralement une recherche d'acceptation et d'affection d'un être cher » (SANTOS ; IPPOLITO, 2009, p. 128). Ainsi, bon nombre des enfants victimes d'abus sexuels avaient un faible niveau d'estime de soi et ont généralement grandi dans l'isolement dans leur propre foyer et dans la communauté

RC: 98977

Disponible en: https://www.nucleodoconhecimento.com.br/psychologie-fr/aspectshistoriques 
dans laquelle ils vivent, se considérant comme différents des autres et n'ayant pas d'orientations sexuelles fondamentales.

Dans le but de prévenir, Santos et Ippolito (2009) suggèrent aux écoles de promouvoir la sensibilisation des membres de la famille responsables de l'éducation des enfants et des adolescents, en utilisant la créativité de leurs éducateurs pour travailler avec ces familles, en les encourageant et en leur apprenant à renforcer les enfants et les adolescents contre les abus sexuels; maintenir une relation de confiance avec l'enfant; y consacrer du temps, et écouter ouvertement ce que l'enfant a à dire, sans banaliser dans les cas où l'enfant maltraité « brise le silence », en pensant qu'il doit inventer les faits et ; construire un réseau de soutien social parmi les membres de la famille au travail pour protéger l'enfant lorsque le membre de la famille qui est responsable de l'éducation du plus jeune doit quitter la maison. Les auteurs déclarent que : « La plupart des actes de violence intrafamiliale et extrafamiliale se produisent lorsque l'enfant est seul avec des jeunes et des adultes chez lui ou chez des connaissances » (SANTOS; IPPOLITO, 2009, p. 129).

La deuxième approche, selon Brino et Willians (2005), est la secondaire, qui vise à détecter les enfants à risque tôt, empêchant ainsi la répétition d'actes de violence, en travaillant à réduire le stress causé par le processus dans le système juridique que l'enfant traverse, considérant que la posture des professionnels travaillant avec l'enfant peut adoucir ou finir par produire des dommages psychologiques à la victime. La troisième et dernière approche présentée par les auteurs est le tertiaire; il vise à surveiller la victime ainsi que l'agresseur, par des professionnels formés, afin de prévenir ou d'atténuer les pertes causées par des abus sexuels, ainsi que d'éventuelles séquelles à long terme.

Compte tenu de l'importance de travailler à la prévention des abus sexuels, d'agir avec les responsables et/ou avec l'enfant, il est possible de planifier des interventions visant la prévention primaire et secondaire. Les mères, par exemple, peuvent jouer un rôle décisif dans la protection contre cette violence. « Lorsqu'ils

$\mathrm{RC}: 98977$

Disponible en: https://www.nucleodoconhecimento.com.br/psychologie-fr/aspectshistoriques 
sont incapables de reconnaître l'occurrence d'abus sexuels et de protection, l'enfant peut devenir plus vulnérable aux abus " (BRINO; WILLIANS, 2005, p. 176). D'où l'importance de chercher des moyens d'informer les mères sur les abus sexuels afin qu'elles deviennent capables de protéger l'enfant contre la survenue de ce type d'agression, et l'école peut être un lieu stratégique pour une telle action, car c'est une institution capable de promouvoir des interventions tirant parti du contact direct avec les parents, enfants et adolescents.

Brino et Williams (2005) renforcent l'idée que donner aux enfants et aux mères les moyens de reconnaître les signes d'abus sexuels peut être une action qui empêche efficacement la survenue de ladite violence. Cette intervention destinée à l'enfant comprendrait la reconnaissance des signes d'approche d'un agresseur, ainsi que la reconnaissance de comportements sexuels inappropriés. Pour les mères, l'action impliquerait de reconnaître les comportements qui sont des indicateurs de maltraitance réussie. Lorsque la proposition de l'action est la détection d'enfants et d'adolescents à risque, la rupture avec des actes violents ou leurs répétitions, l'intervention dans des cas déjà confirmés et l'emploi dans la prévention d'éventuelles séquelles, cette perspective correspond à la prévention secondaire ou même tertiaire.

En plus de la prévention, il est d'une importance fondamentale que nous sachions quoi faire si des abus sexuels ont déjà eu lieu. II faut savoir dénoncer, prendre soin de la victime en adant le soutien nécessaire et en se référant à des traitements spécialisés (BRINO ; WILLIAMS, 2003b, p. 2).

L'une des façons de signaler, comme indiqué sur le site Web de l'UNICEF[7], est de contacter le Conseil de tutelle, considérant qu'il incombe aux conseillers de garantir les droits des enfants et des adolescents. Lors de la réception de la notification, il appartient à ces professionnels d'analyser l'origine de chaque cas, en rendant visite à la famille. Si cela est confirmé, le Conseil renvoie la situation au ministère public.

RC: 98977

Disponible en: https://www.nucleodoconhecimento.com.br/psychologie-fr/aspectshistoriques 
Un autre moyen de dénonciation signalé par l'UNICEF est de contacter les tribunaux de l'enfance et de la jeunesse, dans le cas des municipalités qui n'ont pas le Conseil de tutelle. Les plaintes peuvent également être déposées auprès des postes de police de protection de l'enfance et de l'adolescent et des postes de police pour femmes. II existe également une application pour smartphones et tablettes appelée Proteja Brasil, qui montre les téléphones et l'emplacement de l'institution spécialisée la plus proche de celle du lanceur d'alerte. En outre, l'application offre une assistance en cas de doutes sur le type de violence.

Composez le 100 - Droits de l'homme est un canal par lequel « les plaintes peuvent être anonymes ou, à la demande du plaignant, la confidentialité de la source de l'information est garantie » (BRASIL, s/d). De cette façon, n'importe qui peut déposer une plainte sans être identifié. Dénoncer n'est pas synonyme d'accuser, puisque c'est le professionnel spécialisé qui procédera à l'enquête sur les faits. Par conséquent, il est important de souligner qu'il n'est pas nécessaire d'être certain que l'abus sexuel a eu lieu pour le signaler. C'est un acte d'engagement envers la sécurité et le bien-être des enfants et des adolescents, et l'omission est un acte aussi grave que l'abus sexuel lui-même. L'ECA, en un seul alinéa de l'art. 70-B, détermine que

São igualmente responsáveis pela comunicação de que trata este artigo, as pessoas encarregadas, por razão de cargo, função, ofício, ministério, profissão ou ocupação, do cuidado, assistência ou guarda de crianças e adolescentes, punível, na forma deste Estatuto, o injustificado retardamento ou omissão, culposos ou dolosos (BRASIL, 1990).

Le Statut souligne donc la responsabilité des personnes, qui sont en quelque sorte impliquées dans la vie de l'enfant et de l'adolescent, de porter plainte en cas de violation de leurs droits. Cela réaffirme l'obligation de dénoncer un acte aussi grave qu'un abus sexuel, même s'il s'agit d'un soupçon.

RC: 98977

Disponible en: https://www.nucleodoconhecimento.com.br/psychologie-fr/aspectshistoriques 
Compte tenu de ces notes, il est entendu que les abus sexuels sont un problème grave, avec une histoire large, qui imprègne les discussions politiques, les valeurs culturelles (qui changent selon les régions du monde et avec le moment historique), et pour les problèmes de santé physique, psychologique et sociale, qui peuvent sérieusement compromettre la vie de nombreux enfants et adolescents.

\section{CONSIDÉRATIONS FINALES}

Cet article visait à aborder les aspects historiques des abus sexuels, en passant en revue la législation brésilienne et en explorant les complexités du sujet, même en ce qui concerne la définition et la nomenclature.

On a vu que les enfants et les adolescents n'étaient pas toujours considérés comme des êtres humains qui avaient besoin d'un respect et d'un soin spécifiques et que, tout au long de l'histoire, divers types de violence ont été commis contre ce public sans punition pour les agresseurs, pas même l'attention des autorités publiques pour protéger ces personnes.

Face à plusieurs cas de cruauté et de négligence, des lois étaient instituées en faveur des enfants et des adolescents et, au Brésil, l'ECA marque la mise en place de plusieurs droits, prévoyant la protection des mineurs, et obligeant les adultes à être responsables de leur prise en charge.

Ainsi, les abus sexuels sont l'un des phénomènes qui ont attiré l'attention de l'État et, actuellement, des mesures de protection contre de telles violences ont été établies. Des études montrent les dommages biopsychociaux que cette agression peut causer à la victime et à sa famille, ainsi que les mécanismes qui impliquent la pratique d'abus sexuels, permettant de prendre des stratégies de prévention et d'identifier et de punir les agresseurs de différents profils.

Néanmoins, on sait que la population générale manque encore de connaissances sur le sujet, y compris les membres de la famille et les institutions responsables de la

RC: 98977

Disponible en: https://www.nucleodoconhecimento.com.br/psychologie-fr/aspectshistoriques 
protection des enfants et des adolescents comme l'école, par exemple. De plus, le manque de clarification sur ce phénomène est un facteur qui corrobore que cette violence continue d'être reproduite. II y a donc la nécessité de plus de recherches et d'interventions qui fournissent à la population de plus en plus d'informations sur le sujet, en sensibilisant à l'engagement de tous en faveur de la garantie des droits des enfants et des adolescents, renforçant ainsi la lutte contre les abus sexuels.

\section{RÉFÉRENCES}

ADED et al. Abuso sexual em crianças e adolescentes: revisão de 100 anos de literatura. Revista de Psiquiatria Clínica 33 (4); 204-213, 2006. [online]. Disponível em: <http://www.hcnet.usp.br/ipq/revista/vol33/n4/204.html>. Acesso em: 27 de mai. de 2015.

ARAÚJO, M. de F. Violência e Abuso Sexual na Família. Psicologia em Estudo, Maringá, v. 7, n. 2, p. 3-11, jul./dez. 2002. [online]. Disponível em: <http://www.scielo.br/scielo.php?pid=S1413-

$73722002000200002 \&$ script=sci_arttext>. Acesso em: 09 de set. de 2015.

AZAMBUJA, M. R. F. de. Violência sexual intrafamiliar: é possível proteger a criança? Revista Virtual Textos \& Contextos, oㅡ 5, nov. 2006. [online]. Disponível em: $<$ http://revistaseletronicas.pucrs.br/ojs/index.php/fass/article/view/1022>. Acesso em 09 de set. de 2015.

BRASIL. Ministério da Saúde. Notificação de maus-tratos contra crianças e adolescentes pelos profissionais de saúde. Secretaria de Assistência à Saúde. Série A. Normas e Manuais Técnicos; n. 167. Brasília-DF, 2002. [online]. Disponível em: $<$ http://bvsms.saude.gov.br/bvs/publicacoes/notificacao_maustratos_criancas_adoles centes.pdf>. Acesso em: 27 de mai. de 2015.

. Site: Conselho Nacional de Saúde. 18 de maio - Dia nacional de combate ao abuso e exploração sexual de crianças e adolescentes. s/d. Disponível em

RC: 98977

Disponible en: https://www.nucleodoconhecimento.com.br/psychologie-fr/aspectshistoriques 
<http://conselho.saude.gov.br/ultimas_noticias/2009/15_mai_caesca.htm>.

Acesso em: 06 de jun. de 2016.

Constituição (1988). Constituição da República Federativa do Brasil: promulgada em 5 de outubro de 1988. [online]. Disponível em: <http://www.planalto.gov.br/ccivil_03/Constituicao/Constituicao.htm>. Acesso em: 07 de out. de 2016.

. Estatuto da Criança e Adolescente. Lei 8.069, de 13 de julho de 1990. Brasília, 1990. [online]. Disponível em: $<$ http://www.planalto.gov.br/ccivil_03/LEIS/L8069.htm>. Acesso em 09 de set. de 2015.

Superior Tribunal de Justiça. Súmula 593. O crime de estupro de vulnerável se configura com a conjunção carnal ou prática de ato libidinoso com menor de 14 anos, sendo irrelevante eventual consentimento da vítima para a prática do ato, sua experiência sexual anterior ou existência de relacionamento amoroso com o agente. Terceira Seção. Julgado em 25/10/2017. DJe 06/11/2017. Disponível em $<$ https://www.stj.jus.br/sites/portalp/lnicio>. Acesso em: 14 de abr. de 2021.

BRINO, R. F.; WILLIAMS, L. C. Capacitação do educador acerca do abuso sexual infantile. Interação em Psicologia, 7(02), p. 1-10, 2003b. [online]. Disponível em:<file:///C:/Users/Usuario/Downloads/3218-6348-1-PB\%20(1).pdf>. Acesso em 13 de abr. de 2016.

. Prevenção primária, secundária e terciária do abuso sexual infantil. In:Sobre o comportamento e cognição- Expondo a variabilidade. Org. GUILHARDI, H. J.; AGUIRRE, N. C. de. p.174-181, 2005.

DECLARAÇÃO UNIVERSAL DOS DIREITOS HUMANOS, Adotada e proclamada pela resolução 217 A (III) da Assembléia Geral das Nações Unidas em 10 de dezembro de 1948. Representação da UNESCO no Brasil. Brasília, 1998. [online]

RC: 98977

Disponible en: https://www.nucleodoconhecimento.com.br/psychologie-fr/aspectshistoriques 
Disponível em: <http://unesdoc.unesco.org/images/0013/001394/139423por.pdf>. Acesso em: 05 de jun. de 2016.

FALEIROS, E. T. S; CAMPOS, J. de O. C. Repensando os conceitos de violência, abuso e exploração sexual de crianças e de adolescentes. CECRIA / MJ-SEDH-DCA / FBB / UNICEF. Brasília, 2000. [online] Disponível em: $<$ http://escca.luizaugustopassos.com.br/wpcontent/uploads/2011/02/livro_repensando_os_conceitos_eva_publicacoeshttpwww.mpes_.gov_.branexoscentros_apoioarquivos1.pdf1.pdf>. Acesso em: 02 de jun. de 2016.

GUERRA, A. M. A. et al. Crianças e adolescentes têm direitos: conheça o Sistema de Garantia dos Direitos e saiba como participar. Org. GRACIANI, M. S. S. et al. 1a ed. São Paulo: CONDECA: Manufatura de ideias, 2013.

HABIGZANG, L. F. et al. Abuso sexual infantil e dinâmica familiar: aspectos observados em processos jurídicos. Psicologia: Teoria e Pesquisa, Brasília, v. 21, n.3, p.341-348, $2005 . \quad$ Disponível em:<http://www.scielo.br/scielo.php?script=sci_arttext\&pid=S0102$37722005000300011 \&$ Ing=en\&nrm=iso >. Acesso em: 07 de set. de 2016.

HABIGZANG, L. F.; RAMOS, M. da S.; KOLLER, S. H. A Revelação de Abuso Sexual: As Medidas Adotadas pela Rede de Apoio. Psicologia: Teoria e Pesquisa Out-Dez 2011, Vol. 27 n. 4, pp. 467-473. [online]. Disponível em: <http://www.scielo.br/pdf/ptp/v27n4/10.pdf>. Acesso em: 09 de set. de 2015.

HARMS, M. (org.) Vade Mecum RT. [Equipe RT]. 12 ed. revista, atualizada e ampliada. São Paulo: Editora Revista dos Tribunais, 2016. ISBN 978-85-203-6714-8.

INOUE, S. R. V.; RISTUM, M. Violência sexual: caracterização e análise de casos revelados na escola. Estudos de Psicologia (Campinas) [online]. 2008, vol.25, n.1, pp. 11-21. ISSN 1982-0275. Disponível em: 
<http://www.scielo.br/scielo.php?pid=S0103-

166X2008000100002\&script=sci_abstract\&tIng=pt>. Acesso em: 27 de mai. de 2015.

LEMOS, F. C. S. O Estatuto da Criança e do Adolescente no Brasil atual. Revista Psicologia Política, São Paulo, v. 8, n. 15, p. 93-106, jun. 2008. Disponível em <http://pepsic.bvsalud.org/scielo.php?script=sci_arttext\&pid=S1519549X2008000100007\&lng=pt\&nrm=iso $>$. Acesso em:08 de jun. de 2016.

MINAYO, M. C. S. O significado social e para a saúde da violência contra crianças e adolescentes. In:WESTPHAL, M. F. (org). Violência e criança. São Paulo: Edusp, 2002.

OLIVEIRA, I. S. de. Trajetória Histórica do Abuso Sexual Contra Criança e Adolescente. UniCEUB: Brasília. 2006. Professor-orientador: Dr. Maurício Neubern.

PADILHA, M. da G. S. Abuso sexual contra crianças e adolescentes: considerações sobre os fatores antecedentes e sua importância na prevenção. In: GUILHARDI, H. J.; AGUIRRE, N. C. (Orgs.) Sobre Comportamento e Cognição: Expondo a variabilidade. Santo André: ESETec, 2002, v.10, p. 209 a 220.

PAPALIA, D.; OLDS, S.W.; FELDMAN, R. D. Desenvolvimento Humano. 8 ed.Porto Alegre: Artmed, 2006.

PRADO, M. do C. de A.; CARNEIRO, T. F. Abuso sexual e traumatismo psíquico. Interações, São Paulo, v. 10, n. 20, p. 11-34, dez. 2005. [online] Disponível em:<http://pepsic.bvsalud.org/scielo.php?script=sci_arttext\&pid=S1413$29072005000200002 \&$ lng=pt\&nrm=iso>. Acesso em: 23 de abr. de 2016.

SANTOS. B. R. dos; IPPOLITO, R - Childhood Brasil. O papel da escola no enfrentamento da violência sexual. In: LAVARELLO, F. (Coord). A defesa de crianças e adolescentes vítimas de violências sexuais. São Paulo: Cromosete, 2009, p. $125-130$.

RC: 98977

Disponible en: https://www.nucleodoconhecimento.com.br/psychologie-fr/aspectshistoriques 
SANTOS, V. A. dos; COSTA, L. F.; GRANJEIRO, I. A. C. L. Intervenção no abuso sexual intrafamiliar: ingerência invasiva ou proteção devida? Psico, Porto Alegre, PUCRS, v. 40, n. 4, pp. 516-524, out./dez. 2009. [online]. Disponível em: <http://revistaseletronicas.pucrs.br/ojs/index.php/revistapsico/article/view/4009>. Acesso em: 09 de set. de 2015.

SERAFIM, A. de P. et al. Dados demográficos, psicológicos e comportamentais de crianças e adolescentes vítimas de abuso sexual. Revista de Psiquiatria Clínica, São Paulo, v. 38, n. 4, p. 143-147, 2011. [online]. Disponível em: $<$ http://www.scielo.br/scielo.php?script=sci_arttext\&pid=S010160832011000400006>. Acesso em: 09 de set. de 2015.

UNICEF. Situação mundial da infância (edição especial): celebrando 20 anos da Convenção sobre os Direitos da Criança. UNICEF, 2009. [online] Disponível em: $<$ http://www.unicef.org/brazil/pt/sowc_20anosCDC.pdf>. Acesso em: 03 de jun. de 2016.

VEGA, L. B. da S.; PALUDO, S. dos S. Exploração sexual e rede de proteção na perspectiva da vítima. Arquivos Brasileiros de Psicologia, Rio de Janeiro, v. 67, n. 2, p. 47-60, 2015.

Disponível em

<http://pepsic.bvsalud.org/scielo.php?script=sci_arttext\&pid=S180952672015000200005\&lng=pt\&nrm=iso >. Acesso em: 29 de maio de 2016.

WAISELFISZ, J. J. Atendimento por Violência No SUS. In: Mapa da Violência 2012: Crianças e Adolescentes do Brasil. 1 ed. Rio de Janeiro: CEBELA, 2012. p.62-73.

WILLIAMS, L. C. A. Abuso sexual infantil. In: GUILHARDI, H. J.; AGUIRRE, N. C. (Orgs.). Sobre Comportamento e Cognição: Expondo a variabilidade. Santo André: ESETec, 2002, v.10, p.155-164.

RC: 98977

Disponible en: https://www.nucleodoconhecimento.com.br/psychologie-fr/aspectshistoriques 


\section{ANNEXE - NOTE DE BAS DE PAGE DE RÉFÉRENCE}

3. Données obtenues en https://agenciapatriciagalvao.org.br/violencia/violenciasexual/tres-criancas-ou-adolescentes-sao-abusadas-sexualmente-no-brasil-a-cadahora/

4. Site web du Portail Brésil : < http://www.brasil.gov.br/cidadania-ejustica/2015/07/em-1927-o-brasil-ganhou-oprimeiro-codigo-de-menores> .

5. Site web de DUDH: < http://www.dudh.org.br/declaracao/> .

6. Site Web de CRAMI: <http://www.cramicampinas.org.br

7. Site Web de I'UNICEF : $\quad<$ http:

www.unicef.org/brazil/pt/activities_10790.htm="'">.</http:>

Soumis : Mai 2021.

Approbation : Septembre 2021.

RC: 98977

Disponible en: https://www.nucleodoconhecimento.com.br/psychologie-fr/aspectshistoriques 